\title{
DESIGN OF A HIGH POWER SYNCHROTRON RADIATION ABSORBER FOR HERA
}

\author{
M. Bieler*, E. Lohrmann, A. Meseck, G. Nawrath, M. Seidel, F. Willeke, DESY
}

\section{Abstract}

In the framework of the HERA Luminosity Upgrade Project it is foreseen to move the final focus quadrupole magnets of the proton machine $16 \mathrm{~m}$ closer to the interaction region. As the electrons must not be affected by these magnets, the beam pipes for electrons and protons have to be well separated at these magnets. This implies rather sharp bends with bending radii down to $360 \mathrm{~m}$ for the electron orbit in the interaction region. At an electron energy of $30 \mathrm{GeV}$ and a beam current of $58 \mathrm{~mA}$ these bends cause a high level of synchrotron radiation. The synchrotron radiation will be guided through the interaction region and will be absorbed far downstream at low power densities. However, it is unavoidable to stop parts of the synchrotron radiation beam at high linear power densities of up to $2 \mathrm{~kW} / \mathrm{cm}$ rather close to the interaction point where the electron and proton vacuum systems are separated. In order to protect the proton final focus magnet and the septum beam pipe from this synchrotron radiation, a high power synchrotron radiation absorber was designed. This absorber not only has to withstand the heat load of the synchrotron radiation, but it also has to be designed to minimize the backward scattering of the radiation, because these scattered photons might hit sensitive detector components in the interaction region.

This paper will first give an overview of the geometry of the interaction region with special emphasis on the synchrotron radiation. Then the geometrical and thermal requirements for the high power absorber will be described and finally the layout of the absorber will be presented.

\section{THE UPGRADED HERA INTERACTION REGION}

Fig 1 shows magnet positions, some detector components, the beam orbits and the synchrotron radiation fan for the upgraded HERA interaction region North (South will be nearly identical). In the interaction regions West and East, where the beams do not collide, the geometry of the machine will not be changed.

The straight orbit is the proton orbit, the curved orbit that for the electrons (or positrons). The electron final focus magnets (GJ,GI,GO, GG) are common magnets, acting on both beams. In order to compensate for the influence of the electron final focus magnets on the proton orbit for both possible polarities of the electron machine (electrons or positrons), different horizontal positions of the interaction point (IP) for electrons and positrons were chosen. The proton/positron IP will be shifted $8 \mathrm{~mm}$ towards the center

\footnotetext{
*Email: bieler@ desy.de
}

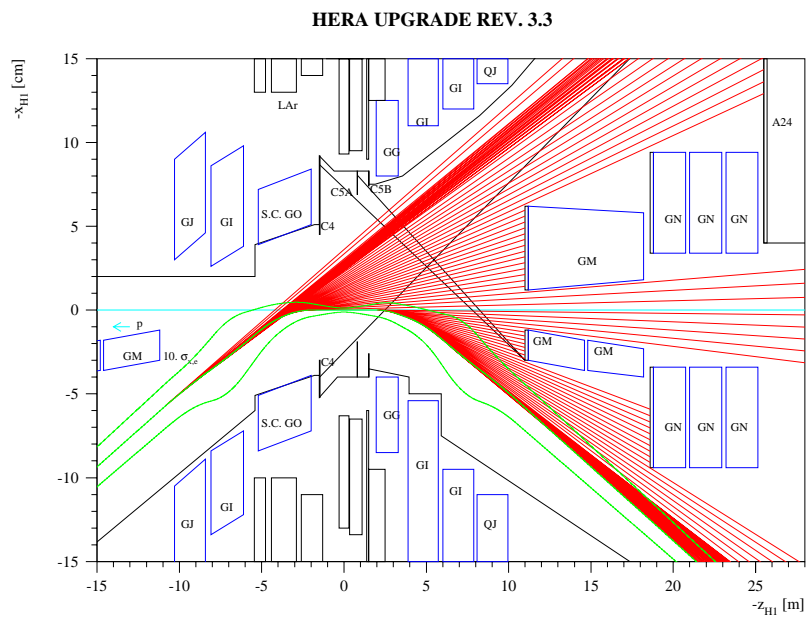

Figure 1: Top view of the upgraded HERA interaction region North with magnet positions, some detector components, the orbits and the synchrotron radiation fan.

of HERA as compared to the proton/electron IP. This results in stronger bending radii for electrons compared to positrons. Therefore in this paper only electron data will be discussed.

Due to the high field strength of the proton final focus magnets (GM, GN), both beam pipes have to be well separated at the first GM magnet. The main difference between the present HERA optics and the Luminosity Upgrade optics is a decrease in spot sizes at the IP, achieved by a 16 $\mathrm{m}$ move of the proton final focus magnets towards the IP. A distance of $11 \mathrm{~m}$ between the interaction point and the beam separation was chosen as a compromise between a small proton spot size in the IP and a tolerable level of synchrotron radiation created by the strong bend of the electrons. More details about the Luminosity Upgrade optics can be found in [1] [2].

\section{SYNCHROTRON RADIATION IN THE INTERACTION REGION}

All numbers given in this paper are calculated for the design current of $58 \mathrm{~mA}$ at the design energy of $30 \mathrm{GeV}$. Table 1 shows the magnets in the interaction region, their bending radii for electrons and the power and the critical energy of the emitted synchrotron radiation.

In order to keep the power density on the synchrotron radiation absorbers and the reflection back into the experimental area low, all absorbers are placed as far from the IP as possible. Between the IP and $11 \mathrm{~m}$ on the right side of the IP there are only emergency absorbers to protect the beam pipe in case of a mis-steared beam. If these absorbers 


\begin{tabular}{|c|c|c|c|}
\hline Magnet & $\begin{array}{c}\text { Bend. Radius } \\
\mathrm{m}\end{array}$ & $\begin{array}{c}\text { Power } \\
\mathrm{kW}\end{array}$ & $\begin{array}{c}\text { Critical Energy } \\
\mathrm{keV}\end{array}$ \\
\hline GJL9 & 2353 & 0.225 & 25 \\
GIL7 & 1863 & 0.358 & 32 \\
GOL2 & 390 & 13.933 & 154 \\
GGR2 & 362 & 6.569 & 166 \\
GIR4 & 402 & 7.683 & 149 \\
GIR7 & 2362 & 0.223 & 25 \\
GJR9 & 1414 & 0.622 & 42 \\
\hline
\end{tabular}

Table 1: Magnets, bending radii, emitted power and critical energy.

are hit by the core of the synchrotron radiation fan, the electron beam will be dumped automatically.

On the right side at $11 \mathrm{~m}$ from the IP, right before the first GM half quadrupole, the beam pipes for electrons and protons are separated. Another beam pipe for the synchrotron radiation from the magnets GJ, GI and GO (left side of the IP) is added on the outer side of the proton beam pipe. In this beam pipe the main part of the synchrotron radiation from the IP is absorbed at low power densities in two absorbers at $19 \mathrm{~m}$ and $25 \mathrm{~m}$ from the IP.

At $11 \mathrm{~m}$ from the IP, where the beam pipe splits from one pipe into three, the sections between the beam pipes have to be protected by a high power synchrotron radiation absorber. Fig. 2 shows the cross section of the three beam pipes behind the absorber (looking away from the IP). The distance between the electron pipe and the proton pipe is given by the width of the mirror plates of the GM half quadrupoles, whereas the distance between the proton pipe and the synchrotron radiation pipe is given by the width of a NEG pump, pumping the proton pipe inside the GM magnets. In order to protect the proton pipe inside the GM magnets from synchrotron radiation, the aperture of the absorber is slightly smaller than the aperture of the beam pipe ( $3 \mathrm{~mm}$ on the outer side, $1 \mathrm{~mm}$ on the inner side).

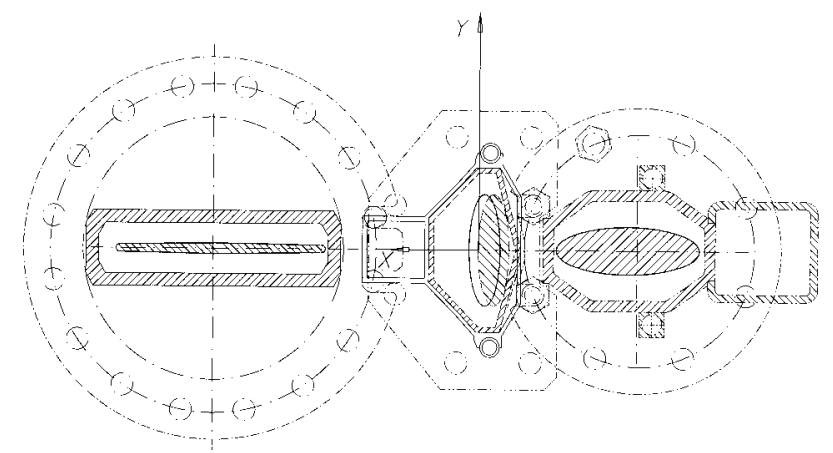

Figure 2: Cross sections of the three beam pipes behind the $11 \mathrm{~m}$ synchrotron radiation absorber (looking away from the IP). Left to right synchrotron radiation pipe, pump channel, proton pipe, electron pipe and pump channel.

\section{THE HIGH POWER ABSORBER}

The synchrotron radiation absorber at $11 \mathrm{~m}$ consists of two parts, the outer part between the synchrotron radiation pipe and the proton pipe and the inner part between the proton pipe and the electron pipe. Table 2 shows the dimensions, positions, beam dimensions and synchrotron radiation power densities for both parts of the $11 \mathrm{~m}$ absorber.

Table 2: Synchrotron radiation power at $11 \mathrm{~m}$

\begin{tabular}{|c|c|c|}
\hline & $\begin{array}{c}\text { Inner } \\
\text { part }\end{array}$ & $\begin{array}{c}\text { Outer } \\
\text { part }\end{array}$ \\
\hline Distance to IP & $11 \mathrm{~m}$ & $11 \mathrm{~m}$ \\
Absorber width $(\mathrm{L})$ & $2 \mathrm{~cm}$ & $4 \mathrm{~cm}$ \\
Line power density & $2 \mathrm{~kW} / \mathrm{cm}$ & $1.1 \mathrm{~kW} / \mathrm{cm}$ \\
Total power & $4 \mathrm{~kW}$ & $4.4 \mathrm{~kW}$ \\
Beam height $(\sigma)$ & $0.8 \mathrm{~mm}$ & $1.3 \mathrm{~mm}$ \\
Absorber area (L $2 \sigma)$ & $0.32 \mathrm{~cm}^{2}$ & $1.04 \mathrm{~cm}^{2}$ \\
Power density (perpend.) & $12.5 \mathrm{~kW} / \mathrm{cm}^{2}$ & $4.23 \mathrm{~kW} / \mathrm{cm}^{2}$ \\
Power density (40 mrad) & $0.5 \mathrm{~kW} / \mathrm{cm}^{2}$ & $0.17 \mathrm{~kW} / \mathrm{cm}^{2}$ \\
\hline
\end{tabular}

The outer part of the absorber has to withstand a line power density of $1.1 \mathrm{~kW} / \mathrm{cm}$, the inner part $2.0 \mathrm{~kW} / \mathrm{cm}$. The height of the synchrotron radiation fan is $\sigma=1.3 \mathrm{~mm}$ on the outer part and $\sigma=0.8 \mathrm{~mm}$ on the inner part. With these numbers and the geometry of the absorber one ends up with power densities which would, at perpendicular incidence of the radiation, destroy the absorber. In order to reduce the heat load on the absorber surface, the surface area exposed to the synchrotron radiation has to be increased by tilting the absorber surface with respect to the incoming radiation. This also helps to minimize the number of photons backscattered from the absorber. These photons can be scattered into the detectors and give a major contribution to the detector background rate. The optimal solution is a vertical tilt of the absorber surface by $40 \mathrm{mrad}$ with respect to the incoming radiation.

The longitudinal dimension of the absorber is limited to about $300 \mathrm{~mm}$, given by the available space between the GJ and GM magnets.

Vertically the absorber has to cover an area $10 \mathrm{~mm}$ above and below the nominal beam position in order to take care for all possible tilts and offsets of the electron beam.

All these geometrical requirements can best be met by an inverse wedge shaped absorber as sketched in fig. 3 .

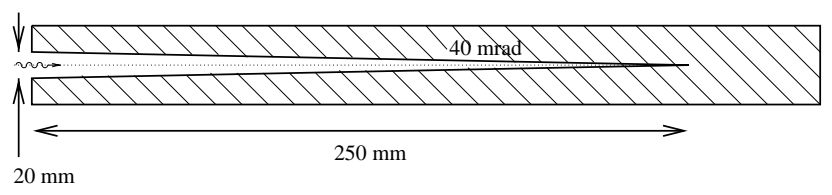

Figure 3: Sketch of the shape of the $11 \mathrm{~m}$ synchrotron radiation absorber (synchrotron radiation coming from the left)

Calculations with the program EGS [3] show that the number of photons reflected back from the absorber to- 
wards the IP (and the detector) can be reduced by a factor 2 by coating the copper body of the absorber with layers of gold $(0.5 \mathrm{~mm})$, silver $(0.5 \mathrm{~mm})$ and again copper $(0.04 \mathrm{~mm})$. However, if the final copper layer gets damaged, the reflection from the inner layers will be much higher than from an uncoated absorber. Therefore the absorbers will first be built uncoated.

Fig 4 shows a horizontal cut through the absorber. The synchrotron radiation fan will hit the tilted surfaces between the beam pipes. Below the three beam pipes five cooling water channels are sketched. The distance between the absorber surface and the water channels, the number and the size of the water channels have to be calculated to minimize the absorber surface temperature without boiling the cooling water.

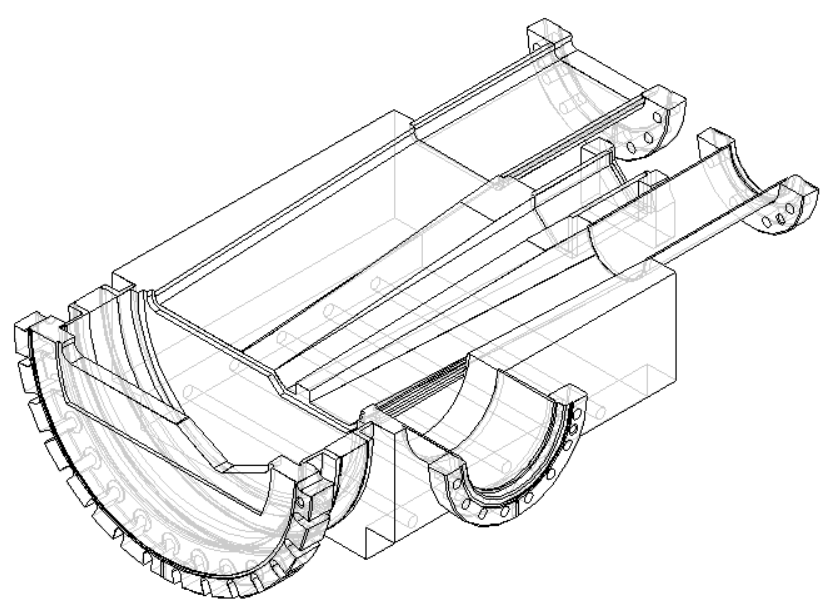

Figure 4: Horizontal cut through the high power absorber.

In the horizontal plane perpendicular to the beam pipes there is a pumping channel with a flange for a vacuum pump attached to the electron pipe. At the position of this pumping channel the wedge of the absorber is still relatively open, so that the proton pipe is also pumped through this channel.

To estimate the temperature distribution in the absorber analytically, a simplified model was considered, where the cooling is done by a plain cooling surface at constant temperature. The difference in temperature $T_{c}$ between the cooling surface and the surface of the absorber is given by

$$
\begin{gathered}
T_{c}(x)=\lim _{\Delta y \rightarrow 0} \frac{P}{L \cdot k} \frac{1}{4 \sqrt{2} \pi^{3 / 2}} \\
\int_{-\infty}^{\infty} e^{-\xi^{2} / 2 \sigma^{2}} \ln \frac{\left(2 y_{0}-\Delta y\right)^{2}+(x-\xi)^{2}}{\Delta y^{2}+(x-\xi)^{2}} d \xi,
\end{gathered}
$$

where $P$ is the incident power, $L$ the horizontal length of the absorber, $k$ the thermal conductivity of the material (copper), $\sigma$ the width (i.e.standard deviation) of the radiation fan, $x$ the distance from the center of the radiation fan, and $y_{0}$ the distance between absorber surface and cooling plane. Assuming the following power and dimensions of: $P=4 \mathrm{~kW}, L=2 \mathrm{~cm}, \sigma=0.8 / 0.04=20 \mathrm{~mm}$ using a
$40 \mathrm{mrad}$ tilt angle, and $y_{0}=110 \mathrm{~mm}$ (distance between the absorber and the cooling channel), the resulting temperature is: $T_{c}=260,280$ and $310^{\circ} \mathrm{C}$ for $\sigma=2,1.5$ and $1 \mathrm{~cm}$. The heat transfer into the cooling water of a pipe is given by: $P=\frac{k_{w} \cdot N_{m} \cdot A}{D} \cdot \Delta T$, where $k_{w}=0.6 \mathrm{~W} / \mathrm{mK}$ is the thermal conductivity of water, $D$ and $l$ the diameter and length of the pipe and $A=\pi D l$ its surface area. $\Delta T$ is the temperature difference between the cooling water and the wall, required to transfer heat with power $P$ into the water.

For a pipe, the heat transfer coefficient (Nußelt number) is given by: $N_{m}=0.032 R e^{0.8} P_{r}^{0.37}\left(\frac{D}{l}\right)^{0.054}$. The Reynolds number $R e$ is $R e=\frac{v D}{\nu}$, where $v$ is the velocity, $\nu$ the viscosity, and $P_{r}$ Prandtl's number $\left(P_{r}=7\right)$.

In order to get a small temperature difference, $R e$ has to be large, requiring a large flow velocity of the water.

The following numerical values have been chosen: $v=$ $3 \mathrm{~m} / \mathrm{s}, \nu=10^{-6} \mathrm{~m}^{2} / \mathrm{s}, D=10 \mathrm{~mm}, R e=30,000$, $l=2 \mathrm{~cm}$ and $P=4 \mathrm{~kW}$. One has to stay below a power density of $100 \mathrm{~W} / \mathrm{cm}^{2}$, and therefore an area of at least $40 \mathrm{~cm}^{2}$ for the cooling tubes is required. One could try 9 tubes arranged in two rows, with a distance of $20 \mathrm{~mm}$ between the centers of the tubes. Putting in numbers one gets $N_{m}=0.032 \cdot 30000^{0.8} \cdot 2.05 \cdot 0.96=240$ and

$$
\Delta T=\frac{P \cdot D}{k_{w} \cdot N_{m} \cdot A}=\frac{4000 \cdot 0.01}{0.6 \cdot 240 \cdot 40 \cdot 10^{-4}}=69^{\circ} \mathrm{C}
$$

Assuming a temperature of the cooling water of $30^{\circ} \mathrm{C}$, the temperature at the hottest spot of the absorber will be about $30+69+280 \approx 380^{\circ} \mathrm{C}$. The rise in water temperature will be comparatively small, about $1^{\circ} \mathrm{C}$.

For comparison numerical calculations have also been carried out with a finite element code. Here the surface temperature of the absorber is of the order of $350^{\circ} \mathrm{C}$ and the temperature on the surface of the cooling water pipes is well below $100^{\circ} \mathrm{C}$.

\section{CONCLUSION}

The HERA Luminosity Upgrade requires a high power synchrotron radiation absorber near the interaction region. The proposed design fulfills the geometric requirements, given by the surrounding magnets and vacuum chambers, minimizes the amount of back-scattered radiation and reduces the thermal load on the absorber surfaces.

\section{REFERENCES}

[1] U. Schneekloth (editor), "The HERA Luminosity Upgrade", DESY-HERA-98-05, (1998).

[2] M. Seidel , "Luminosity Upgrade of HERA", these proceedings.

[3] L. Suszycki, "Synchrotron Radiation at the HERA Upgrade", ZEUS Note 98-039, (1998). 